\title{
Determinação das propriedades físicas da madeira de Astronium lecointei Ducke
}

As variações de umidade e da densidade do lenho das árvores são as principais causas dos defeitos de secagem, como o empenamento e fendilhamento das peças de madeira. A umidade de equilíbrio deve ser determinada para o local onde a madeira será empregada. Este trabalho teve como objetivo a caracterização tecnológica da madeira de Astronium lecointei Ducke, definindo dessa maneira as aplicações adequadas para a espécie. A espécie apresentou 0,815 $\mathrm{g} / \mathrm{cm}^{3}$ de densidade, densidade aparente seca de $0,726 \mathrm{~g} / \mathrm{cm}^{3}$, sendo maior que a densidade básica, que apresentou o valor de $0,658 \mathrm{~g} / \mathrm{cm}^{3}$. 0 inchamento linear da madeira de Astronium lecointei Ducke foi de $0,126 \%, 1,294 \%$ e $0,475 \%$, correspondendo respectivamente a longitudinal, tangencial e radial e 0 inchamento linear Astronium lecointei Ducke apresentou Anisotropia dimensional de inchamento (Ai) =0,553 e Anisotropia dimensional de contração (Ac) $=0,568$. A madeira de Astronium lecointei Ducke apresentou valores de densidade que a caracterizam como uma madeira de média densidade. Além disso, apresentou menores valores para o inchamento linear e para a contração volumétrica quando comparada a outras madeiras amazônicas.

\section{Determination of physical properties of wood Astronium lecointei Ducke}

\begin{abstract}
Variations in humidity and wood density are the main causes of drying defects, such as warping and cracking of wood pieces. The equilibrium humidity must be determined for the place where the wood will be used. This work aimed at the technological characterization of Astronium lecointei Ducke wood, thus defining the appropriate applications for the species. The species showed $0.815 \mathrm{~g} / \mathrm{cm}^{3}$ of density, dry bulk density of $0.726 \mathrm{~g} / \mathrm{cm}^{3}$, being higher than the basic density, which presented a value of $0.658 \mathrm{~g} / \mathrm{cm}^{3}$. The linear swelling of Astronium lecointei Ducke wood was $0.126 \%, 1.294 \%$ and $0.475 \%$, corresponding to longitudinal, tangentia and radial and linear swelling, respectively. Astronium lecointei Ducke presented dimensional swelling anisotropy $(\mathrm{Ai})=0.553$ and dimensional contraction anisotropy $(\mathrm{Ac})=0.568$. The Astronium lecointei Ducke wood presented density values that characterize it as a medium density wood. In addition, it showed lower values for linear swelling and volumetric contraction when compared to other Amazonian woods.
\end{abstract}

Keywords: Contraction; Swelling; Properties of Wood.

Topic: Desenvolvimento, Sustentabilidade e Meio Ambiente

Reviewed anonymously in the process of blind peer.
Received: 06/05/2020

Approved: $29 / 06 / 2020$
Sarah Stephanie Rebelo Traian Baumann (iD) Universidade Federal do Oeste do Pará, Brasil http://lattes.cnpq.br/7722661512593219

http://orcid.org/0000-0003-1916-1552

sarah.enflor@gmail.com

Camila Amorim Santa Brigida (D)

Universidade Federal do Oeste do Pará, Brasil http://lattes.cnpq.br/7618034411950250

http://orcid.org/0000-0003-1229-7422

brigida88camila@gmail.com

Lívia Karine Lima Rabelo (iD

Universidade Federal do Oeste do Pará, Brasil

http://lattes.cnpq.br/2513116920954057

http://orcid.org/0000-0002-7294-6423

liviarabello16@gmail.com

\author{
Mayra Piloni Maestri \\ Universidade Federal Rural da Amazônia, Brasil \\ http://lattes.cnpq.br/2687102042811310 \\ http://orcid.org/0000-0002-8936-952X \\ mayrapmaestri@hotmail.com \\ Marina Cardoso de Aquino (iD \\ Universidade do Estado de Santa Catarina, Brasil \\ http://lattes.cnpq.br/2168843028631934 \\ http://orcid.org/0000-0002-0160-0804 \\ marinaacardosoo@gmail.com
}

\section{Referencing this:}

BAUMANN, S. S. R. T.; BRIGIDA, C. A. S.; RABELO, L. K. L.; MAESTRI, M. P.; AQUINO, M. C.. Determinação das propriedades físicas da madeira de Astronium lecointei Ducke. Nature and Conservation, v.13, n.3, p.122-128, 2020. DOI: http://doi.org/10.6008/CBPC23182881.2020.003.0012 


\section{INTRODUÇÃO}

A Muiracatiara (Astronium lecointei Ducke), pertence à família Anacardiaceae, tem uma larga distribuição na região Amazônica nos estados do Acre, Amapá, Maranhão, Mato Grosso, Pará, Rondônia. A árvore da Muiracatiara caracteriza-se por ser de grande porte, com altura comercial variando de 15 a $20 \mathrm{~m}$ e diâmetro de $60 \mathrm{~cm}$ e ser pouco frondosa, possui madeira muito pesada, cerne de cor variável do bege-rosado ao castanho-escuro-avermelhado, possui alta resistência mecânica e baixa retratabilidade (LOUREIRO et al., 2000; LORENZI, 2002; NAHUZ, 2013).

Atualmente o uso da madeira de Muiracatiara está sendo cada vez maior, pois a mesma possui excelente acabamento e pintura e é de fácil trabalhabilidade. Devido a essas características, grande parte dessa matéria-prima é absorvida pelo setor da construção civil na fabricação de assoalhos, móveis decorativos, indústrias de embalagens e de painéis (NAHUZ, 2013). Porem Jankowsky (1990) afirma que se esta madeira for seca ao ar, pode apresentar problemas de empenamentos e rachaduras, e se a secagem artificial desta madeira for muito drástica, poderão ocorrer rachaduras profundas e endurecimento superficial, afetando a qualidade final dos produtos originados e sua correta utilização.

Para atender as necessidades do setor madeireiro, as pesquisas, nos últimos anos, vêm buscando aprimorar a qualidade da matéria-prima. As principais propriedades físicas da madeira que refletem nas características final do produto são a densidade básica e a aparente, a umidade e a estabilidade dimensional/contração, sendo a primeira característica a que melhor expressa a qualidade da madeira, com os constituintes anatômicos, por facilitar a medição e pelo rendimento volumétrico e gravimétrico (SHIMOYAMA et al., 1989; TRUGILHO et al., 1990).

Um dos fatores limitantes para a utilização de novas espécies florestais, principalmente as espécies amazônicas, é a falta de dados sobre suas propriedades e, consequentemente, de seus possíveis usos comerciais e industriais. Dessa forma, tornam-se imprescindíveis estudos com a finalidade de definir as aplicabilidades em que as mesmas serão destinadas, pois determinadas espécies podem ser vetadas para uma finalidade onde a estabilidade dimensional seja um fator primordial (GALVÃO et al., 1985). Pela importância da determinação das propriedades físicas da madeira, o presente trabalho teve como objetivo a caracterização tecnológica da madeira de Astronium lecointei Ducke, com o nome vernacular de Muiracatiara, definindo dessa maneira as aplicações adequadas para a espécie.

\section{MATERIAIS E MÉTODOS}

O experimento foi realizado no Laboratório de Tecnologia de Produtos Florestais, da Universidade Federal Rural da Amazônia, UFRA. A mensuração das Propriedades Físicas da Madeira (PFM) foi efetuada através de ensaios de laboratório, utilizando equipamentos próprios a essa finalidade e seguindo normas que especificam os métodos, procedimentos, fórmulas de cálculo, formas e dimensões de corpos de prova, etc.

A Astronium lecointei Ducke foi adquirida na empresa madeireira SEMASA, que se encontra no bairro da Pratinha em Belém. Os fustes das árvores foram coletados no município de Uruará, latitude 03느이" sul 
e a uma longitude 5344'12" oeste, pertencente ao estado do Pará, onde a empresa coloca em prática seu plano de manejo.

A metodologia utilizada baseou-se na Norma MB 1269/79 da Associação Brasileira de Normas Técnicas - ABNT. Para a análise das propriedades físicas da madeira, foram utilizados 15 (quinze) corpos de prova (CP) com dimensões aproximadas de $2 \times 3 \times 5 \mathrm{~cm}$ (tangencial, radial e longitudinal, respectivamente). Inicialmente, as amostras foram lixadas, a fim de corrigir imperfeições, identificadas e, posteriormente, obteve-se o peso úmido ( $\mathrm{Pu}$ ), com balança analítica, e o volume úmido, através de medições com paquímetro de precisão 0,005 mm, nas três faces dos corpos de prova úmidos (Longitudinal, Transversal e Radial) $L$ (tang), $L($ rad) $L(\log )$ e Vol úmido. Em seguida, as amostras foram imersas em água destilada até que atingissem a completa saturação em bomba a vácuo, a fim de recuperar a umidade perdida após o corte e então, retirouse o volume do corpo saturado através do método de pesagem baseado no princípio de Arquimedes e realizou-se as devidas medições com paquímetro.

Após a determinação do volume saturado do corpo de prova, este foi submetido à secagem em uma estufa com $103^{\circ} \pm 2^{\circ} \mathrm{C}$ de temperatura até atingir a estabilização do peso em quatro pesagens consecutivas, condição em que caracteriza a ausência completa de água na parede celular, correspondente ao peso do corpo de prova absolutamente seco (Po) ( $0 \%$ de umidade) e, novamente, realizaram-se medições com paquímetro nas três faces do corpo de prova seco. A medição da umidade relativa foi realizada pelo higrômetro e consistiu em 10 medições para cada escala de 1-20, sendo que foi considerada a escala que apresentou o valor médio mais próximo do valor determinado pelo método da pesagem. Foram calculadas as seguintes variáveis:

Teor de Umidade (TU): É a relação entre o peso de água contido em seu interior e o seu peso em estado completamente seco, expresso em porcentagem, calcula-se utilizando a seguinte fórmula:

$$
\mathrm{TU}=[(\mathrm{Pu}-\mathrm{Po}) / \mathrm{Po}] * 100
$$

Onde:

$U(\%)=$ Teor de umidade em \%

$\mathrm{Pu}=$ Peso úmido da madeira em gramas

$\mathrm{Po}_{\mathrm{o}}=$ Peso seco da madeira em gramas

Densidade aparente úmida $\boldsymbol{\rho}_{(u \%)}$ : A determinação da densidade de uma amostra de madeira pode ser realizada determinando-se seu peso e seu volume utilizando-se a fórmula:

$$
\rho(U \%)=\mathrm{Pu} / \mathrm{V}_{(\mathrm{U})}
$$

Onde:

$\mathrm{Pu}=$ Peso úmido

$\mathrm{V}(\mathrm{U})=$ Volume úmido

Densidade aparente seca $\rho_{(0 \%)}$ : Densidade a $0 \%$, definida como a relação entre massa e volume obtidos para $\mathrm{U}=0$, onde a massa e o volume são obtidos após secagem em estufa $103 \pm 2^{\circ} \mathrm{C}$ (Rezende et al, 1988) utiliza-se a seguinte fórmula:

$$
\rho_{(0 \%)}=P_{(0 \%)} / V_{(0 \%)}
$$

Sendo:

$\mathrm{P}(0 \%)=$ peso a $0 \%$ de umidade

$V_{(0 \%)}=$ Volume a $0 \%$ de umidade

Inchamento linear máximo: O máximo inchamento linear de uma madeira é dado pela diferença 
entre suas dimensões 'L' em estado saturado (PSF) e suas dimensões em estado absolutamente seco.

$$
\alpha_{L(\max )}=\frac{L_{(P S F)}-L_{(0)}}{L_{(0)}} \times 100
$$

Onde:

Lmáx = máximo inchamento linear (\%).

$L_{P S F}=$ dimensão $L$ da madeira em estado saturado $(\mathrm{cm} 3)$;

$L_{0}=$ dimensão $L$ da madeira em estado seco $(\mathrm{cm} 3)$.

Inchamento Volumétrico Máximo: O máximo inchamento volumétrico de uma madeira é dado pela diferença entre suas dimensões volumétricas em estado saturado (PSF) e suas dimensões em estado absolutamente seco.

$$
\alpha_{L(\max )}=\frac{V_{(P S F)}-V_{(0)}}{V_{(0)}} \times 100
$$

Onde:

Vmáx = máximo inchamento volumétrico (\%). $V_{P S F}=$ volume da madeira em estado saturado $(\mathrm{cm} 3) ;$ $V_{0}=$ volume da madeira em estado seco $(\mathrm{cm} 3)$.

Contração Volumétrica Máxima: A contração volumétrica máxima foi calculada de acordo com a expressão:

$$
\beta_{(V \max )}=\frac{V_{P S F}-V_{0}}{V_{P S F}} x 100
$$

Onde: Vmáx = máximo inchamento volumétrico (\%). $V_{P S F}=$ volume da madeira em estado saturado $(\mathrm{cm} 3) ;$ $V_{0}=$ volume da madeira em estado seco $(\mathrm{cm} 3)$.

Anisotropia da Madeira: $O$ fator de anisotropia dimensional (A), de contração (Ac) ou de inchamento (Ai), é expresso pela relação entre os movimentos lineares radial e tangencial, como expresso pelas equações:

$$
\begin{aligned}
& A i=\text { (tang) } / \text { (rad) } \\
& A C=(\text { tang }) /(\text { rad })
\end{aligned}
$$

\section{RESULTADOS E DISCUSSÃO}

\section{Teor de umidade}

Observou-se com a comparação das médias dos valores do método de pesagem com os valores médios obtidos em cada uma das 20 escalas do higrômetro e concluiu-se que a média da escala 13 do higrômetro foi a que mais se aproximou apresentando $15,56 \%$ de umidade em relação à média dos valores obtidos pelo método de pesagem que foi de $21,66 \%$ de umidade.

\section{Densidade aparente úmida, densidade aparente seca e densidade básica aparente}

Notou-se que a presença de água nos poros dos corpos de prova influenciou no aumento da densidade aparente úmida, que é a relação entre o peso e volume úmido dos corpos de prova, apresentando 
$0,815 \mathrm{~g} / \mathrm{cm}^{3}$ de densidade. Já a densidade aparente seca ( $0 \%$ de umidade) foi $0,726 \mathrm{~g} / \mathrm{cm}^{3}$, sendo maior que a densidade básica, que apresentou o valor de $0,658 \mathrm{~g} / \mathrm{cm}^{3}$.

Resultados semelhantes foram encontrados para a madeira de Cedro-marinheiro (Guarea trichilioides L.), que também é uma espécie amazônica, onde a densidade a $12 \%$ de umidade encontrada foi $0,7604 \mathrm{~g} / \mathrm{cm}^{3}$, a densidade aparente seca no valor de $0,7061 \mathrm{~g} / \mathrm{cm}^{3}$ e a densidade básica de $0,6124 \mathrm{~g} / \mathrm{cm}^{3}$ (LOGSDON et al., 2008).

Se comparada a outras madeiras amazônicas, pode ser classificada como madeira de media densidade, pois seus resultados são próximos aos encontrados por Silveira et al. (2012) que assim classificaram as espécies: Parkia paraensis $\left(0,561 \mathrm{~g} / \mathrm{cm}^{3}\right)$; Brosimum parinarioides $\left(0,588 \mathrm{~g} / \mathrm{cm}^{3}\right)$; Clarisia racemosa $\left(0,665 \mathrm{~g} / \mathrm{cm}^{3}\right)$ e Ocotea rubra $\left(0,720 \mathrm{~g} / \mathrm{cm}^{3}\right)$. Levando em consideração a classificação dada por Carvalho (1996) para as folhosas, a madeira de Astronium lecointei Ducke se encontra dentro do intervalo proposto para as madeiras moderadamente pesadas. A madeira da Muiracatiara apresenta densidade superior à madeira de Paricá (Schizolobium amazonicum Herb), pois segundo os resultados de Almeida et al. (2013) as densidades básica e aparente determinadas foram de $0,30 \mathrm{~g} / \mathrm{cm}^{3}$ e $0,37 \mathrm{~g} / \mathrm{cm}^{3}$.

\section{Inchamento linear e inchamento linear máximo}

Segundo Carvalho (1996), inchamento é o quociente expresso em percentagem, da variação em um sentido definido na dimensão de um corpo-de-prova durante o acréscimo do teor de umidade da madeira, relacionado à dimensão no estado seco. É a alteração da dimensão 'I' em um sentido definido, durante o acréscimo do teor de umidade da madeira de U1 para U2.

O inchamento linear da madeira de Astronium lecointei Ducke foi de 0,126\%; 1,294\%; 0,475\%; correspondendo respectivamente a longitudinal, tangencial e radial e o inchamento linear máximo foi 0,290\%; 6,367\%; 3,437\%, longitudinal, tangencial e radial, respectivamente. O inchamento foi considerado pequeno, de baixa resistência à umidade.

Os valores encontrados no presente estudo podem ser considerados baixos se comparado à pesquisa de Menezes (2014), que encontraram resultados de 10,58\% (radial); 9,05\% (tangencial); 7,59\% (longitudinal) para a madeira de Eucalyptus saligna e 8,0\% (radial); 5,52\% (tangencial); 6,52\% (longitudinal) para a madeira de Corymbia citriodora.

\section{Contração volumétrica máxima e inchamento volumétrico máximo}

Segundo Rezende et al. (1988) a variação em volume na madeira se processa no intervalo de umidade entre $0 \%$ a $28 \%$, aproximadamente, (até o PSF), sendo a madeira praticamente estável, com pequenas variações volumétricas, para umidades acima deste valor. A espécie em questão apresentou valor de inchamento volumétrico máximo de 10,34\% e contração volumétrica máxima de 9,37\%. Valores aproximados a estes foram encontrados para Pinus elliottii no estudo de Balloni (2009), onde foi observado o inchamento volumétrico de 10,89\% e a contração volumétrica máxima de 9,82\%.

Comparando-se as outras espécies amazônicas, a Astronium lecointei Ducke apresentou menor 
contração volumétrica pois, segundo a pesquisa de Cardoso et al. (2012) Swartzia laurifólia apresentou $16,86 \%$ de contração volumétrica total, Nectandra dioica apresentou 13,02\% e Qualea dinizii apresentou $11,85 \%$.

\section{Anisotropia}

O coeficiente de anisotropia dimensional pode ser utilizado para qualificar a madeira quanto aos defeitos provenientes da secagem. Esse coeficiente pode ser obtido tanto a partir de ensaios de inchamento, quanto de retração. De maneira geral, quanto mais baixo for o Índice de Retração Anisotrópica (IRA) que é a razão entre as retrações tangenciais (RT) e radiais (RR) melhor é uso em marcenaria, logo, a mesma é indicada para tal fim, pois da madeira de Astronium lecointei Ducke apresentou Anisotropia dimensional de inchamento $(\mathrm{Ai})=0,553$ e Anisotropia dimensional de contração $(\mathrm{Ac})=0,568$. As madeiras que apresentam coeficiente de anisotropia até 1,50 são indicadas para a fabricação de objetos que necessitem de menores variações em suas dimensões, como móveis finos, instrumentos musicais, aparelhos de esporte, entre outros.

\section{CONCLUSÕES}

A madeira de Astronium lecointei Ducke apresentou valores de densidade que a caracterizam como uma madeira de média densidade. Apresentou menores valores para o inchamento linear e para a contração volumétrica quando comparada a outras madeiras amazônicas. Para o coeficiente de anisotropia, por apresentar valores baixos ( $\mathrm{A} i=0,553$ e $\mathrm{Ar}=0,568)$ concluímos que a espécie em estudo possui características excelentes para fabricação de objetos que necessitem de menores variações em suas dimensões, como móveis finos, instrumentos musicais, aparelhos de esporte, entre outros.

\section{REFERÊNCIAS}

ALMEIDA, D. H.. Caracterização completa da madeira da espécie amazônica Paricá (Schizolobium amazonicum) em peças de dimensões estruturais. Revista Árvore, v.37, n.6, p. 1175-1181, 2012.

BALLONI, C. J. V.. Caracterização física e química da madeira de Pinus Elliottii. Engenharia Industrial Madeireira Itapeva, 2009.

CARDOSO, C. C.; MOUTINHO, V. H. P.; ANDRADE, F. W. C.; SOUSA, M. R.; SOUSA, L. K.. Caracterização tecnológica da madeira de três espécies amazônicas visando à comercialização. In: ENCONTRO BRASILEIRO EM MADEIRAS E EM ESTRUTURAS DE MADEIRA, 13. Anais. Vitória: Universidade Federal do Espírito Santo, 2012.

CARVALHO, A.. Madeiras Portuguesas: Estruturas anatómica. Lisboa, 1996.

JANKOWSKY, I. P.. Madeiras Brasileiras. Caxias do Sul: Spectrum, 1990.

LOGSDON, N. B.; FINGER, Z.; PENNA, E. S.. Caracterização físico-mecânica da madeira de Cedro-marinheiro, Guarea trichilioides L. (Meliaceae). Scientia Florestais, Piracicaba, v.36, n.77, p.43-51, 2008.

LORENZI, H.. Árvores brasileiras: manual de identificação e cultivo de plantas arbóreas nativas do Brasil. 4 ed. Nova Odessa: Instituto Plantarum, 2002.

LOUREIRO, A. A.; SILVA, M. F.; ALENCAR, J. C,. Essências madeireiras da Amazônia. Manaus: INPACPPF, 2000.

MENEZES, W. M.. Modificação térmica nas propriedades físicas da madeira. Ciência Rural, v.44, n.6, p.1019-1024, 2014.

NAHUZ, A. R.; MIRANDA, M. J. A. C.; IELO, P.; PIGOZZO, R. J. B.; YOJO, T.. Catálogo de madeiras brasileiras para a construção civil. São Paulo: Instituto de Pesquisas Tecnológicas do Estado de São Paulo, 2013.

SHIMOYAMA, V. R.; BARRICHELO, L. E. G.. Influência de características anatômicas e químicas sobre a densidade básica da madeira de Eucalyptus spp. In: CONGRESSO ANUAL DE CELULOSE E PAPEL. Anais. São Paulo: ABTCP, 1991. 
SILVEIRA, L. H. C.; REZENDE, A. V.; VALE, A. T.. Teor de umidade e densidade básica da madeira de nove espécies amazônicas. Acta Amazônica, v.43, n.2, 2012.
TRUGILHO, P. F.; SILVA, D. A.; FRAZÃO, F. J. L.; MATOS, J. L. M.. Comparação de métodos de determinação da densidade básica em madeira. Acta Amazônica, v.20, p.307-319, 1990.

A CBPC - Companhia Brasileira de Produção Científica (CNPJ: 11.221.422/0001-03) detém os direitos materiais desta publicação. Os direitos referem-se à publicação do trabalho em qualquer parte do mundo, incluindo os direitos às renovações, expansões e disseminações da contribuição, bem como outros direitos subsidiários. Todos os trabalhos publicados eletronicamente poderão posteriormente ser publicados em coletâneas impressas sob coordenação da Sustenere Publishing, da Companhia Brasileira de Produção Científica e seus parceiros autorizados. Os (as) autores (as) preservam os direitos autorais, mas não têm permissão para a publicação da contribuição em outro meio, impresso ou digital, em português ou em tradução. 